corpus luteum supposed to be formed after conception. Diseases arrest the development of the vesicles, and it is in this arrest that the true cause of amenorrhoea is in many cases to be sought; for the catamenial flow is the result of the sanguineous congestion of the internal generative organs, by which the development of the vesicle in its highest degree is accompanied. The state of the ovaries is so intimately connected with the general health of an individual, that the internal appearance of these organs after death is said by Raciborski to be alone adequate to determine whether the patient has sunk under an acute or a chronic disease, or whether she has latterly menstruated regularly.

Phenomena analogous to those specified above as occurring in the human fernale at the menstrual period, are remarked also in animals during their rutting season. Among these the ovarian vesicles are found to increase gradually in size during the interval between the rutting epochs; and they ultimately escape altogether at these epochs without congress of the male. 'The spontaneous detachment of the human ovum at the end of the menstrual epoch naturally renders that period the most favourable for impregnation, and readily yields a reason for the fact that conception is most commonly referred to that epoch by pregnant women. "Ofl5 women (says Dr. R.) who specified accurately the period of their latest menstruation, as well as the dates of the connubial act, 5 evidently conceived from coitus taking place from two to four days previous to the period at which the catamenia was due. In 7 , conception dated from coitus occurring two or three days after menstriation; in 2 , it took place at the actual period of the catamenia; and in one only so long as ten days after the latter had disappeared.

Considered with respect to her generative function, wornan holds a place intermediate between rutting animals, which are capable of impreynation only at fixed seasons of the year, and those animals in which a coitus only is required to prodnce impregnation at any season. She, however, approaches much nearer in point of this analogy to the former class, her power of reproduction being infinitely the more active at her menstrual periods, to which the rutting time in brutes bears a strict physiological resemblance.-Gaz. des Hôpitaux.

** There is much that is new, rational, and striking in the above views, and we shall keep an eye upon any future observations that may be made by Dr. Raciborski on this subject.

Analysis of a Polfous.-M. Girardin, of Rouen, has found a vaginal polypus which he submitted to a chemical examination, to consist chiefly of fibrin, with small quantities of fatty matter, hematosine, and phosphate of lime.

\section{CASE OF ABSCESS}

IN

\section{THE WALLS OF THE UTERUS,}

COMMUNICATING WITH THE RECTUM.

Related at the Westminster Medical Society, Dec. 3, 1842, by Dr. Fredertc Bird.

Mrs. G., aged 37, had, previously to the last three year, enjoyed general good health, menstruating regularly. At this date she married, and was soon afterwards attacked with acute deep-seated pain in the hypogastric region, radiating to all parts of the pelvis, and increased by micturition and defecation. These symptoms were associated with general constitutional disturbance, and, in fact, with all the ordinary symptoms of inflammation affecting the uterus. She passed through the usual forms of treatment, and although the more urgent symptoms were mitigated, yet she continued to suffer during the three following months from occasional pain in the region of the uterus, always produced by attempts at expelling the contents of the bladder or rectum, the discharge of fæces being also sometimes effected with great difficulty. An internal examination made at this period detected the uterus lower in the vagina than usual; there existed marked enlargement of that organ, the chief increase in size being found to occupy the posterior wall; the os and cervix uteri were painful to the touch and tumid.

Shortly after the raginal examination had been made about half an ounce of pus suddenly escaped from the rectum, and she experienced immediate relief from her former symptoms. She now became the subject of diarrhoea, generally passing from six to eight evacuations daily, each of which contained more or less purulent matter; pain in micturition was no longer felt, but she invariably suffered greatly when passing motions. The diarrhcea could not be arrested by any of the remedies employed; her general health, nevertheless, slowly improved, and she went into the country, where she remained during the succeeding two years, little or no variation in her symptoms having occurred. The diarrhoea, and with it the discharge of pus from the rectum, continued; on some occasions more than a pint of pus has been thus evacuated during twenty-four hours, and she observed that whenever the pus failed to be discharged so freely as nsual the local pain became aggravated. During the whole of this period menstruation had been very irregular, generally occurring at intervals of eight or nine days, accompanied by much lumbar pain and the passage of coagula.

After the lapse of the time mentioned she again applied to Dr. Bird, suffering from nearly all her previous symptoms, and, in addition to them, profuse menorrhagia; the 
pain in the region of the uterus was extremely acute, increased by the passage of the fæces and by pressure on the lower part of the abdomen, to which became added a neuralgic condition of the genital organs, the slightest pressure upon which produced extreme suffering; so great was the pain thus excited that she was accustomed to employ a mechanical contrivance to prevent the bedclothes from touching the pubes. A vaginal examination was, with much difficulty and pain, again made; the uterus was found to be nearly in the same state as before, excepting that it had become quite immoveabie, appearing as if impacted in the pelvis, just as may be observed in some forms of malignant disease affecting that organ. No benefit resulted from inedical treatment, occasional relief only being afforded by large doses of opium and the external application of belladonna.

She continued to suffer from frequent discharges of blood from the vagina, and from all her former symptoms, until the lapse of six weeks, when she sank exhausted by the extreme suffering produced by her disease.

A post-mortem examination was made twenty-four hours after death. On laying open the abdomen the, omentum, small intestines, and all the pelvic viscera, were found agglutinated together by peritoneal adhesions of old date. On raising the uterus it was seen to be firmly attached by its upper and posterior portion to the rectum; it presented an irregular form, having the fundus enlarged to about thrice its natural size. A longitudinal section showed this enlargement to have been produced by an abscess seated in the substance of the wall of the fundus uteri, the cavity of which contained about an ounce of dark thick pus; the walls of the abscess varied in thickness from one to three-quarters of an inch, the thinnest portion being nearest to the cavity of the uterus. A communication by means of a short sinus could be traced passing from the cavity of the abscess to the adherent portion of the rectum, and opening into that intestine by an aperture sufficiently large to admit of the passage of a thick probe, and evidently of old formation. No communication existed between the uterine cavity and that of the abscess. The os and cervix presented no evidence of malignant disease. The Fallopian tubes and ovaries were adherent to the uterus, and could with difficulty be distinguished. The uterus had never been impregnated.

He (Dr. B.) had been induced to bring the case before the notice of the society chiefly from the rarity of such forms of disease; very few cases had as yet been lescribed, and those which he had hitherto met with in the works of Madame de Boivin and others were complicated with carcinoma or other malignant disease of the uterus. In the instance he had related no evidence of such disease existed, but it was clearly a case of infiammation of the substance of the uterus terminating in the formation of $a b$ scess. It was difficult to account for the neuralgic state of the generative organs, unless the immoveable and apparently impacted condition of the uterus might be received as sufficient to produce such an effect by mechanically pressing upon the surrounding nerves, an idea favoured by the fact that at the earlier period of the case, when the uterus was not thus fixed, the pain was limited to that viscus and did not extend to the external organs.

* * The preparation was exhibited to the society, where Dr. Chowne stated that he had never seen a similar case. The uterus and its appendages, he said, were in that conglomerated state which is usually observed in women who had led an irregular life, and which was, probably, in them, dependent upon early and excessive excitement. This adhesion of the ovaries, Fallopian tubes, \&c., in prostitutes, had been noticed also in France. Perhaps, he added, in Dr. Bird's case, this condition might have been the result of the disease under which the patient laboured. On the same occasion another member of the society, Dr. Reid, said that he also was unacquainted with the record of any similar case, excepting those related by Madame Boivin, and thought the disease must be a remarkably rare one.

DETECTION OF ARSENIC IN THE EXCRETIONS.

An apothecary of Dresden, named Meurer. had entertained doubts as to the truth of the assertion that urine is the only excretion in which arsenic is to be detected after having been swallowed, and accordingly experimented on the perspiration, nasal mucus, and frees of horses, to which the substance had been purposely administered. In the mucus and perspired matter he has hitherto discovered no trace of the poison; but this circumstance he attributes to his having obtained these excretions in small quantities only. It was otherwise with the fæces, in which arsenious acid was proved to be present, not in a state of mechanical mixture, as if it had passed through the stomach and small intestine unchanged, but in intimate combination with the fæcal matter. According to Meurer the fæces possess an advantage over the urine for the researches of the chemist or medical jurist, in cases where arsenic has been taken, inasmuch as the poisan may be detected in them after a greater lapse of time. Traces of arsenic are not generally demonstrable in the urine for more than four days after its administration; in the faces they may be proved to exist for at least twenty-four hours longer with facility. -Wochensch. fur die gesammte Heilkund.) 